\title{
THE LHC INJECTION KICKER MAGNET
}

\author{
L. Ducimetière, N. Garrel \\ CERN, AB Division, CH-1211 Geneva 23, Switzerland \\ M.J. Barnes, G.D. Wait \\ TRIUMF, 4004 Wesbrook Mall, Vancouver, B.C., Canada V6T 2A3
}

\begin{abstract}
Proton beams will be injected into LHC at $450 \mathrm{GeV}$ by two kicker magnet systems, producing magnetic field pulses of approximately $900 \mathrm{~ns}$ rise time and up to $7.86 \mu$ s flat top duration. One of the stringent design requirements of these systems is a flat top ripple of less than $\pm 0.5 \%$. Both injection systems are composed of 4 travelling wave kicker magnets of $2.7 \mathrm{~m}$ length each, powered by pulse forming networks (PFN's). To achieve the required kick strength of $1.2 \mathrm{Tm}$, a low characteristic impedance has been chosen and ceramic plate capacitors are used to obtain $5 \Omega$. Conductive stripes in the aperture of the magnets limit the beam impedance and screen the ferrite. The electrical circuit has been designed with the help of PSpice computer modelling. A full size magnet prototype has been built and tested up to $60 \mathrm{kV}$ with the magnet under ultra high vacuum (UHV). The pulse shape has been precision measured at a voltage of $15 \mathrm{kV}$. After reviewing the performance requirements the paper presents the magnet design, emphasizing several novel design features, and discusses the test results.
\end{abstract}

\section{INTRODUCTION}

CERN, the European Laboratory for Particle Physics, is constructing the Large Hadron Collider (LHC), which will bring protons into head-on collisions at an energy of $14 \mathrm{TeV}$, and heavy ions at $1148 \mathrm{TeV}$.

The LHC will be located in the $27 \mathrm{~km}$ long tunnel that previously housed the Large Electron Positron Collider (LEP), and be filled at $450 \mathrm{GeV}$ through a chain of injector machines. Two counter-rotating beams, which can collide in up to 4 interaction points, will circulate in two horizontally separated channels. Each beam channel will be filled by 12 batches of protons from the Super Proton Synchrotron (SPS), injected successively along the machine circumference from a dedicated injection point. Injection is carried out in the horizontal plane by means of a septum magnet followed by a vertical fast pulsed kicker system.

\section{INJECTION REQUIREMENTS}

The beam to be injected approaches the kicker system at an angle of $0.8 \mathrm{mrad}$, requiring a total kick strength of 1.2 Tm for deflection onto the central machine orbit. The reflections and the flat top ripple of the field pulse must be lower than $\pm 0.5 \%$, a very demanding requirement, to limit the beam emittance blow-up due to injection oscillations. The pulse repetition time is imposed by the duration of the SPS acceleration cycle which is about $18 \mathrm{~s}$. The LHC will be filled with 12 batches of either $5.84 \mu \mathrm{s}$ or $7.86 \mu \mathrm{s}$ duration each, to be deposited successively on the machine circumference. This leaves 11 gaps of $0.94 \mu$ s to account for the kick rise time and one gap of $3.0 \mu$ s for the kick fall time. The latter gap will also be used for the rise time of the beam dumping kickers. Table 1 summarizes the main parameters of the injection kicker system.

\section{Table 1: System requirements and parameters}

\begin{tabular}{|lrl|}
\hline Number of magnets per system & 4 & \\
Kick strength per magnet & 0.3 & T.m \\
Magnet beam aperture (diameter) & 38 & $\mathrm{~mm}$ \\
Characteristic impedance & 5 & $\Omega$ \\
Operating charging voltage (PFN) & 54 & $\mathrm{kV}$ \\
Field flat top ripple & $< \pm 0.5$ & $\%$ \\
Field flat top duration & up to 7.86 & $\mu \mathrm{s}$ \\
Field rise time $0.5 \%-99.5 \%$ & 0.9 & $\mu \mathrm{s}$ \\
Field fall time $99.5 \%-0.5 \%$ & 3.0 & $\mu \mathrm{s}$ \\
Magnet length & 2.7 & $\mathrm{~m}$ \\
\hline
\end{tabular}

\section{MAGNET DESIGN AND CONSTRUCTION}

\section{General}

A carefully matched high bandwidth system is needed to obtain the stringent pulse response requirements. The system is therefore composed of a multi-cell PFN and a multi-cell travelling wave kicker magnet, connected by a matched transmission line and terminated by a matched resistor. A low system impedance (Z) of $5 \Omega$ has been chosen [1]. Fig. 1 gives the basic circuit diagram.

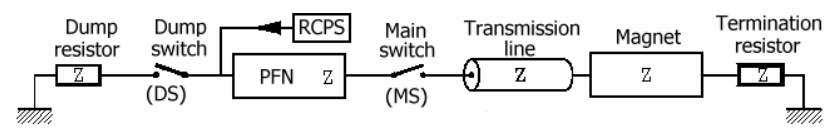

Fig. 1: Schematic circuit of system.

The design voltage is $60 \mathrm{kV}$, as in most SPS kicker installations, allowing the use of several proven components such as transmission line, connectors and termination resistors. The voltage on the magnet is half of the PFN voltage: allowing for overshoot, the design voltage of the magnet is $35 \mathrm{kV}$.

\section{Design strategy}

The ferrite cores of the kicker magnet have a Cconfiguration to allow earthing of the coaxial cable input 
connection and the output connection to the termination resistor. To achieve a characteristic impedance of $5 \Omega$, ceramic plate capacitors with contoured rims have been used inside the magnet. The kicker magnet consists of 33 cells, which is a compromise between the bandwidth and the cost. The cell size allows an optimum filling of the $540 \mathrm{~mm}$ diameter vacuum tanks reused from the LEP separators. The nominal self-inductance and capacitance of each cell is $101 \mathrm{nH}$ and $4.04 \mathrm{nF}$, respectively.

In order to minimize beam impedance, while allowing a fast field rise time, a ceramic pipe with silver stripes on its inner wall is placed within the aperture of the magnet. The stripes provide a path for the image current of the beam and screen the ferrite against induced heating [2]. The stripes are directly connected to the standard vacuum chambers of the machine at one end and via a decoupling capacitance of $300 \mathrm{pF}$ at the other end.

\section{Modeling}

The electrical circuit of the complete kicker system, including PFN, thyratrons, coaxial cables, kicker magnet and terminators has been optimised using PSpice [3]. The voltage dependency of the terminating resistor has also been modelled. The PFNs were optimised to obtain the required rise time, flattop, fall-time and post pulse field quality, first with an ideal transmission line magnet modelled, and then with a realistic model of the kicker magnet including all known parasitic elements. An input RC network and a capacitor in parallel with the terminating resistor were added and optimised to obtain the required field quality.

Some damping is necessary to minimize the pulse ripple. The ferrite has a quality factor (Q) of 5 , but it cannot be considered as being the $\mathrm{Q}$ factor of the cell inductance because the latter is defined by the aperture of the magnet. This has been verified by measurement and damping resistors of about $30 \Omega$ have been connected in parallel with each cell inductance to reduce the effective Q. A more efficient damping would affect field rise time.

The mathematical model also includes beam impedance stripes mutually coupled to the self-inductance of the corresponding cell of the magnet [1]. This allows the effect of the stripes upon the field quality to be included.

The predicted magnetic field for the prototype PFN and prototype kicker magnet constructed at CERN was well within the specification, providing allowance for real pulses. The dump switch was turned on during the simulation to define the duration of the field pulse. The predicted rise-time was approximately $820 \mathrm{~ns}$, and the corresponding fall time $2.2 \mu \mathrm{s}$. The field flat top varied by almost $\pm 0.3 \%$, a result of already known variations in the diameter of the prototype PFN coils [4]. Detailed measurements made on the series of nine PFNs manufactured at TRIUMF [5] have shown that this problem has been overcome.

\section{Construction}

Each of the 33 cells includes 2 ceramic plate capacitors with diameter $210 \mathrm{~mm}$, to arrive at the capacitance of
$4.04 \mathrm{nF}$, including the plate end effect. The capacitors are mounted between the high voltage and ground plates and the connections are ensured by toroidal spring contacts all around the capacitor rims. This electrical contact is critical and depends strongly on a good rim geometry. The high voltage and ground plates are spaced by three ceramic-metal insulators, which together compose an independent cell assembly. Careful design of the mechanical components (distances and radii) aimed at maintaining electrical fields below $5 \mathrm{MV} / \mathrm{m}$.

Fig. 2 shows a schematic cross section of a magnet cell with C-shaped ferrite yoke and 2 ceramic matching capacitors mounted between high voltage and ground plates, and Fig. 3 shows the magnet during assembly.

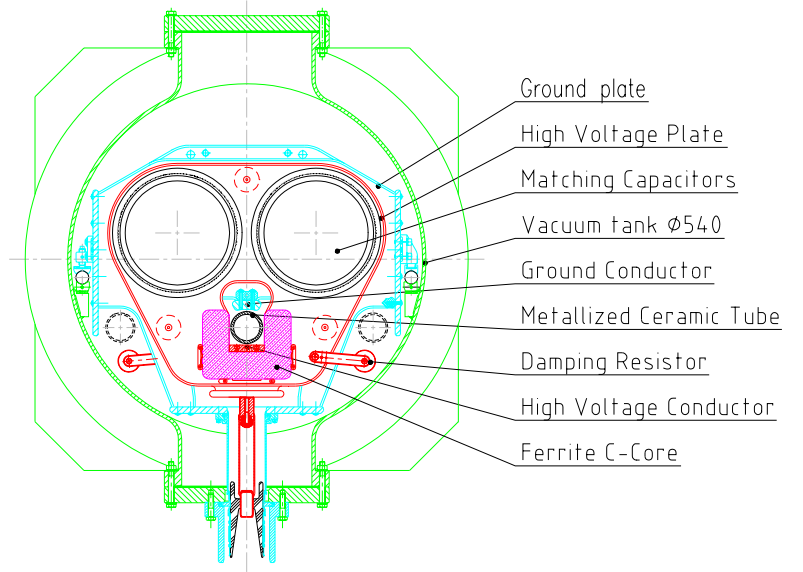

Fig. 2: Kicker magnet cross section

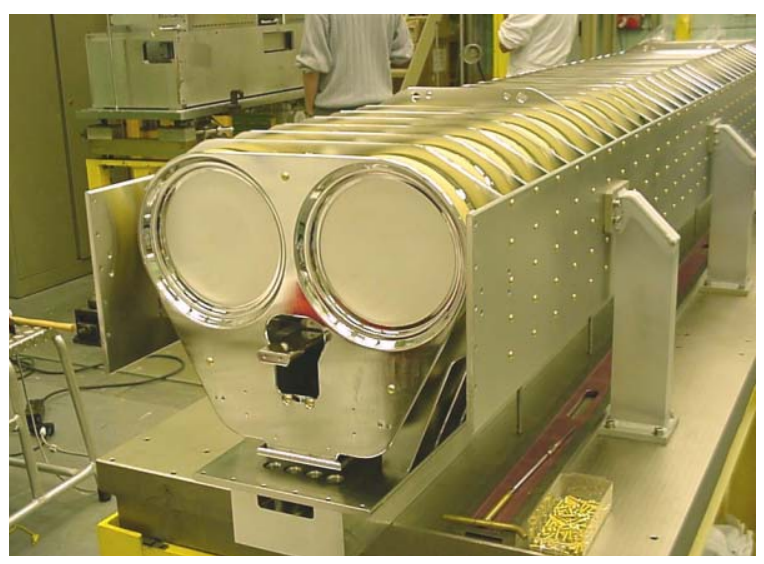

Fig. 3: Prototype kicker magnet during assembly

For stability reasons capacitors are made of class 1 ceramic with relative permittivity between 75 and 90 , consisting of a monolithic ceramic with silver layers for the electrodes. Three manufacturers have been qualified in Europe.

Outgassing tests have shown that these ceramic capacitors can be used in UHV. However, their highvoltage behaviour was unexpectedly poor under vacuum, with surface flash-over problems, calling for challenging development work to achieve a reliable operation at full voltage. Successful test results have finally been obtained 
by improving two important aspects that drastically affect the voltage holding:

a) The geometry of the transition between the connecting plates and the capacitor has been studied in detail with finite element computer codes. Deflector parts have been implemented to eliminate the electrical field at the triple junction; in addition high voltage and ground plates have been electropolished.

b) The vacuum quality has been improved by bake out at $300^{\circ} \mathrm{C}$ and vacuum firing on most of the parts of the magnet. Therefore, the magnet has been designed to be bakeable and made of stainless steel, aluminium being unsuitable for such temperature cycles.

Low inductance damping resistors have been developed for UHV compatibility. They consist of two counterwound KanthalC wires on a ceramic rod.

The ceramic pipe inside the aperture of the magnet is made from a $3 \mathrm{~m}$ long extruded ceramic tube with a wall thickness of $4 \mathrm{~mm}$. The stripes are produced using a printed circuit board technique, but complicated by the access to the inside of the tube. Two layers of silver painting are first applied uniformly inside with bake out at $800^{\circ} \mathrm{C}$ after each layer. The mask is maintained against the inner wall by vacuum while UV light is passed through the pipe. The decoupling capacitor at one end is obtained using the ceramic tube itself as dielectric. The tube extends outside the magnet on which the outer surface is uniformly silver painted over $200 \mathrm{~mm}$ and the inner covered by the stripes, thus giving the capacitance between each stripe and the outer layer. The outer layer is to be connected to the metallic beam tube of the machine.

\section{Test results}

A prototype magnet has been built. The vacuum level after bake out was as low as $3 \cdot 10^{-11}$ mbar. The magnet has been successfully tested with $4 \cdot 10^{5}$ pulses at $60 \mathrm{kV}$ PFN voltage with only two non-destructive breakdowns.

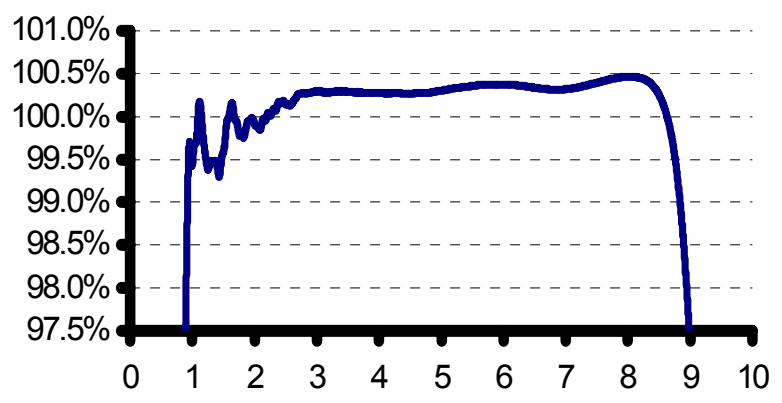

Fig. 4: Measured magnetic field (horiz. scale $\mu$ s)

After these tests in UHV, the magnet has been pulsed in air at reduced voltage $(15 \mathrm{kV})$ for precision magnetic measurements in the aperture. The measurements have been carried out by means of an inductive loop probe with a precision better than $0.1 \%$ [6]. The metallization being not yet realized on the full length ceramic pipe, a metallised mylar film with the final stripe geometry has been installed and connected adequately to represent the final coupling. Fig. 4 shows that the resulting field pulse just fulfils the stringent requirement. The measured rise and fall times, with dump switch triggered, are $880 \mathrm{~ns}$ and $2.7 \mu \mathrm{s}$, respectively.

\section{CONCLUSIONS}

A large and relatively strong fast pulsed magnet has been designed for the LHC injection and constructed for its specific requirements. The simulation results have shown that it is theoretically possible to achieve the required magnetic field pulse. The mechanical design has been complicated by the need to bake-out the full magnet due to the ceramic capacitors. A prototype of the whole system has been built and tested at CERN at full voltage with the magnet under UHV. Although some parasitic effects have not been identified and incorporated in the equivalent circuit, the magnetic field waveform is within specifications.

The design thus validated, the series construction has been launched and most of the components are in production in industry. The injection test in LHC with beam, using 4 kicker magnets, is foreseen in spring 2006.

\section{ACKNOWLEDGEMENTS}

We would like to remember our late colleague John O. Pearce who initiated the mechanical development of this magnet. Many thanks to M. Mayer and B. Riffaud who brought the mechanical design to its final state, to J. Bertin, J. Hurte and J. M. Dury who contributed in the construction and test of the prototype, to E. Carlier and his team who provided the electronics and controls, and to J. H. Dieperink and J. Uythoven for the development of the probe and magnetic measurement method.

\section{REFERENCES}

[1] L. Ducimetière, U. Jansson, G.H. Schröder, E.B. Vossenberg, M.J. Barnes, G.D. Wait, "Design of the injection kicker magnet system for CERN's $14 \mathrm{TeV}$ proton collider LHC', $10^{\text {th }}$ IEEE Int. Pulsed Power Conference, Albuquerque, June 1995, pp 1406-1411.

[2] R.L. Gluckstern, L. Vos, B. Zotter, "Shielding Particle Beams by Thin Conductors", CERN-SL2002-014-AP, May 2002.

[3] Cadence, 2655 Seely Av., San Jose, CA 95134, USA.

[4] M.J. Barnes, G.D. Wait, TRIUMF, E. Carlier, L. Ducimetière, G.H. Schröder, E.B. Vossenberg, "Construction and $60 \mathrm{kV}$ tests of the prototype pulser for the LHC injection kicker system", $12^{\text {th }}$ IEEE Int. Pulsed Power Conference, Monterey, June 1999, pp 777-780.

[5] M.J. Barnes, G.D. Wait, L. Ducimetière, "Low voltage measurements on nine PFNs for the LHC injection kicker systems", $8^{\text {th }}$ EPAC, Paris, June 2002, pp 2520-2522.

[6] J. H. Dieperink, J. Uythoven, “CERN High precision magnetic field measurements of fast pulsed magnets", $12^{\text {th }}$ International Magnet Measurement Workshop, Grenoble, October 2001. 